Rev. Biol. Trop. 52(1): 177-185, 2004

www.ucr.ac.cr www.ots.ac.cr www.ots.duke.edu

\title{
Coexistencia de Lutjanus peru y Lutjanus guttatus (Pisces: Lutjanidae) en la costa de Guerrero, México: relación con la variación temporal en el reclutamiento
}

\author{
Xavier Chiappa-Carrara ${ }^{1}$, Agustín A. Rojas-Herrera² \& Maite Mascaró ${ }^{3}$ \\ 1 Unidad de Investigación en Ecología Marina, FES-Z, Universidad Nacional Autónoma de México. Apdo. Postal 9- \\ 020, 15000 México D.F. Tel.: +52(555)623-0756 ext.114; chiappa@ servidor.unam.mx \\ 2 Escuela Superior de Ecología Marina, Universidad Autónoma de Guerrero. Apdo. Postal 1606, 39390 Acapulco, \\ Guerrero, México. \\ 3 Laboratorio de Ecología y Biología Marina Experimental, Facultad de Ciencias, Universidad Nacional Autónoma de \\ México. Calle 26 \# 1, Playa Norte, 24140 Ciudad del Carmen, Campeche, México.
}

Recibido 21-IX-2001. Corregido 17-III-2003. Aceptado 07-IV-2003.

\begin{abstract}
Monthly volumes of capture of Lutjanus peru and Lutjanus guttatus from the coast of Guerrero, Mexico, were analyzed considering eight annual cycles. Time-series, auto correlation, and cross-correlation analysis showed that monthly abundance of populations display unsystematic variations. The FiSAT software was used to obtain the recruitment patterns of both species, using length-frequency data. Our results support the hypothesis that temporal phase-shifts in reproductive events, hence recruitment, explain the coexistence of these species. The outcome of this mechanism is a temporal succession of specific recruit abundance off the coasts of Guerrero, Mexico. The uncoupling of the recruitment events between these species, induces a separation of recruits; therefore, the intake of a particular set of preys could take place at different times.
\end{abstract}

Key words: coexistence, recruitment, Lutjanus guttatus, Lutjanus peru, Guerrero, Mexico.

El grupo de los lutjánidos está compuesto, en términos generales, por especies que se alimentan de un espectro relativamente amplio de presas y que, cuando están presentes en el mismo hábitat, compiten por los mismos recursos tróficos presentando niveles de traslape dietéticos relativamente altos (Haight et al. 1993, Guevara et al. 1994, Sámora-Zapata y VegaCendejas 1998, Sierra 1997, Vega-Cendejas 1998, Franks y VanderKooy 2000).

Los estudios sobre la alimentación natural de los juveniles del flamenco (Lutjanus guttatus Steindachner 1869) y del huachinango ( $L$. peru Nichols \& Murphy 1922) muestran que existe un gran número de presas comunes que ambas especies utilizan en los primeros estadios de desarrollo (Saucedo-Lozano et al. 1999, Saucedo-Lozano y Chiappa-Carrara
2000). Asimismo, Rojas-Herrera et al. (2004) reportan que existe un gran número de presas comunes a L. peru y L. guttatus en la fase adulta y que existe un mayor grado de afinidad entre las dietas de aquellas especies de lutjánidos que habitan un mismo sitio, que entre organismos de la misma especie presentes en diferentes localidades.

Existen especies con hábitos generalistas de alimentación que presentan patrones de traslape en el uso de los recursos (Paine 1977, Connell 1978). En casos de coexistencia, una especie puede cambiar su comportamiento reproductivo desfasando temporalmente el período de reproducción dando así un alivio a la competencia y permitir la presencia de un competidor potencial. Por ejemplo, la asincronía de las estaciones reproductivas de dos 
mugílidos en el Noroeste del Golfo de México atenúa su competencia (Álvarez-Lajonchere 1976, Ibáñez-Aguirre 1993). De la misma manera, si las especies están sometidas a explotación pesquera, la densidad de las poblaciones puede disminuir de tal forma que disminuya la competencia y puedan coexistir (Jensen 1997).

Por otra parte, el punto de vista clásico establece que la competencia constituye una de las fuerzas más importantes que definen la estructura de una comunidad y para que dos especies puedan coexistir, se requiere de una diferenciación de sus nichos que les permita repartir el recurso limitante (Gause 1934, MacArthur 1958). Por ejemplo, la diferenciación de nichos explica la reducción de la competencia por la limitada disponibilidad de huéspedes en los peces asociados a las anémonas arrecifales (Elliot y Mariscal 2001). Asimismo, ayuda a explicar el carácter multiespecífico de la comunidad de cíclidos del Lago Malawi (Genner et al. 1999). Sin embargo, tanto en estos trabajos como en otros (Butler y Smith 1992, Pfister 1992, Evans y Tallmark 1985, Mullon et al. 2002), se ha sugerido que las variaciones temporales en reclutamiento juegan un papel preponderante en la diversidad de los sistemas y determinan, al menos en parte, los patrones locales de coexistencia.

Un único pulso de reclutamiento puede limitar el tamaño de una población si se constituye como un evento denso-independiente (Sinclair 1989), pero puede regular el tamaño poblacional a partir de mecanismos denso-dependientes, tal como la mortalidad post-reclutamiento (Hassell 1986). La evidencia empírica indica que las fluctuaciones en el reclutamiento están relacionadas con las variaciones en la densidad local (Caley et al. 1996).

En resumen, las especies ecológicamente similares tienden a competir intensamente si están presentes en un mismo ambiente (Dudgeon et al. 1999) y se ha reconocido que la competencia es la principal fuerza que define la estructura de una comunidad (Giller 1984). Como consecuencia de esto, pueden actuar procesos de exclusión competitiva (Gause 1934) o bien de disminución de la intensidad de la competencia. Los procesos que se han descrito para explicar la forma en la que las especies evitan competir actúan en distintas escalas. En progresiones de tiempo grandes, se manifiesta una tendencia evolutiva hacia la especialización divergente relacionada con el uso del recurso por el cual compiten (Margalef 1981); en escalas de tiempo más cortas, las especies similares presentan variaciones espaciales o temporales en la reproducción y el reclutamiento, disminuyendo de esta forma la intensidad de la competencia (Gaines y Roughgarden 1985, Caley et al. 1996) y promviendo la coexistencia (Chesson 1985).

L. guttatus y L. peru son especies residentes y dominantes en el litoral de Guerrero; ambas se capturan de manera persistente a lo largo de todo el año con los mismos artes y en los mismos caladeros, siendo de gran importancia para las pesquerías ribereñas. Dada la abundancia de ambas especies de lutjánidos en la plataforma continental adyacente a Guerrero, México, y considerando que los cambios estacionales en la abundancia de sus presas naturales pueden generar distintos niveles de competencia por el alimento, la pregunta que surge es ¿cuáles son los procesos biológicos que explican su coexistencia? En el presente estudio postulamos que la coexistencia de L. peru y L. guttatus puede estar mediada por las variaciones temporales en el reclutamiento reflejadas en términos de una sucesión temporal de la abundancia de los reclutas de cada especie. El objetivo de este trabajo es analizar las oscilaciones anuales de los volúmenes de captura como una medida de las abundancias de L. peru y L. gutattus en la costa de Guerrero, México, y relacionarlas con las variaciones de los pulsos de reclutamiento para así obtener una estimación indirecta de la intensidad de la competencia entre estas especies.

\section{MATERIAL Y MÉTODOS}

Se realizó un análisis retrospectivo de los volúmenes de captura de L. peru y L. guttatus. Los datos de las capturas provienen de las 
diferentes oficinas de la Secretaría del Medio Ambiente, Recursos Naturales y Pesca (delegación Guerrero). La información se organizó por mes y año considerando el período comprendido entre 1984 y 1994, por ser ésta la serie de datos más completa. Los valores de captura fueron suavizados con la métrica de Henderson, considerando las medias móviles de 5 términos (Visauta-Vinacua 1998), para reducir las irregularidades (fluctuaciones aleatorias) en las series de tiempo. Dado que la autocorrelación en series temporales permite identificar la dependencia entre las observaciones, se llevó a cabo un análisis de este tipo en los periodos anuales considerados, con el objeto de cuantificar la regularidad de la señal, representando así la forma básica del fenómeno de captura.

Dado un conjunto de mediciones $y_{1}, y_{2}, \ldots$, $y_{n}$, correspondientes a los valores mensuales de captura, la función de retraso autocorrelativa se define como (Box y Jenkins 1976)

En este caso, se asume que las observaciones están equidistantes en el tiempo, expresando el valor de asociación entre dos valores de la misma variable (tiempo) en $X_{i}$ y $X_{i+k}$.

Los análisis de correlación cruzada han sido ampliamente utilizados para extraer el patrón de una señal a partir de un conjunto de una serie de datos variables. Asimismo, este tipo de análisis permite identificar los retrasos entre los pulsos de las señales e identificar la periodicidad de la misma (Legendre y Legendre 1998). De esta manera, se realizó este análisis para obtener una medida de la similitud entre las series de datos de captura de cada especie. Dado que el resultado se expresa como un valor de correlación $(\rho)$ entre las dos señales, considerando distintos valores de retraso en el tiempo, fue posible determinar las posiciones de correspondencia de las capturas observadas debidas a retrasos sucesivos en el tiempo.

Dado que en la hipótesis de este trabajo se ha postulado que las variaciones temporales en el reclutamiento de $L$. peru y L. guttatus pueden explicar la coexistencia de estas especies, se obtuvieron los patrones de reclutamiento empleando el modelo propuesto por Gayanilo et al. (1996) resuelto mediante el paquete de programas FiSAT. Este método permite reconstruir dichos patrones a partir de una serie temporal de datos de frecuencia de talla. De esta forma, se retro-proyectaron las estructuras mensuales de talla de cada especie, alimentando el modelo con los parámetros de crecimiento específicos obtenidos por Rojas-Herrera (2001). Este método asume (a) que todos los peces considerados en la muestra crecen conforme a la ecuación definida por los parámetros de crecimiento y (b) que en uno de los doce meses el reclutamiento es cero. Estrictamente, la segunda suposición no se cumple en los peces tropicales, puesto que existen evidencias de que se incorporan periódicamente reclutas al stock. Si bien en este trabajo no se validaron los períodos reproductivos a través de técnicas histológicas, fue posible estimar el número y la magnitud relativa de cada evento de reclutamiento y así obtener información general sobre este proceso (Pauly et al. 1984, Pauly 1987).

\section{RESULTADOS}

La tendencia de las capturas de L. peru muestra fluctuaciones mensuales importantes en los primeros 7 años del período de observación considerado (1984-1994). En enero de 1992 se alcanzó la máxima captura mensual (183 ton), seguida por una caída considerable en febrero (31 ton). A partir de este mes, se observa una disminución en los volúmenes de las capturas. En los últimos 20 meses de este análisis se observó que el volumen de las capturas normalmente es menor a las 80 ton por mes (Fig. 1A).

Las capturas mensuales de L. guttatus fueron, por lo regular, menores a 5 ton (Fig. 1B). Es evidente que, especialmente en los últimos meses analizados, los volúmenes de captura muestran una tendencia positiva al pasar de 2 ton en enero de 1994 a 15.9 ton en diciembre de 1994.

La periodicidad de los picos y de los valles de los volúmenes de captura no es evidente, y existe una gran variación interanual de los 

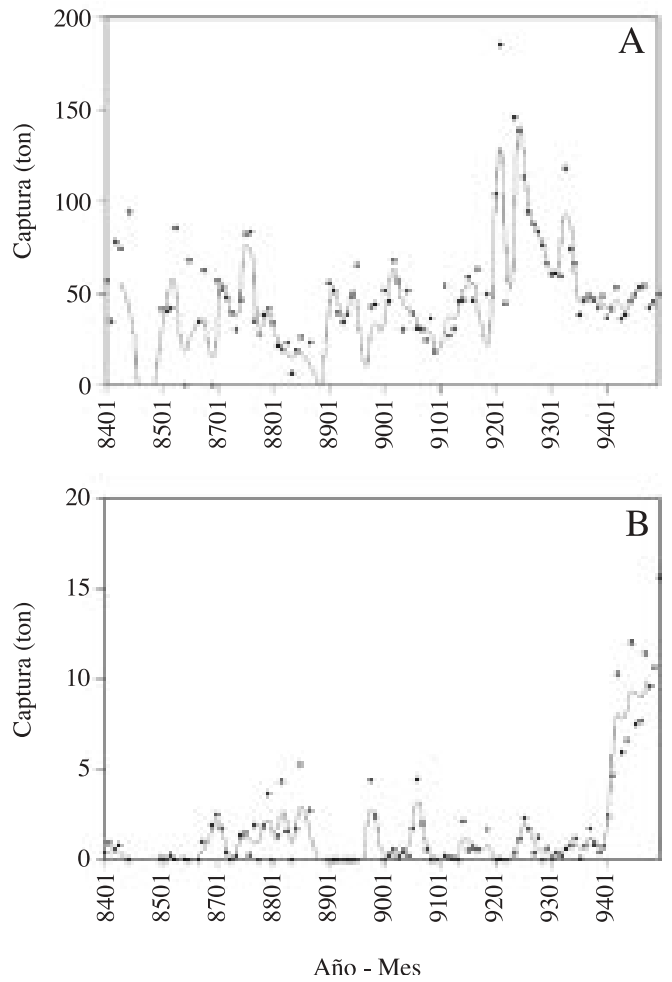

Fig. 1. Series de tiempo (- valores suavizados con la métrica de Henderson, medias móviles de 5 términos) de las capturas mensuales (ton) observadas (•). A: Lutjanus peru. B: Lutjanus guttatus.

Fig. 1. Time-series analysis (- smoothed values using Henderson's weighted moving averages of 5 terms) of observed $(\bullet)$ monthly captures (ton). A: Lutjanus peru. B: Lutjanus guttatus.

datos promedio por mes. En el caso de L. peru (Fig. 2A) los valores más elevados ocurrieron en el verano (julio y agosto, con capturas superiores a 60 ton), con la excepción del pico que se presenta en febrero. En cambio, los valles corresponden a diciembre $(\leq 37.5$ ton). La serie de tiempo de las capturas promedio de $L$. guttatus (Fig. 2B) muestra que los valores máximos ocurren en diciembre ( $\sim 3.8$ ton) en forma más o menos periódica y posteriormente se reducen para alcanzar los valores mínimos durante enero ( $\leq 0.6$ ton).
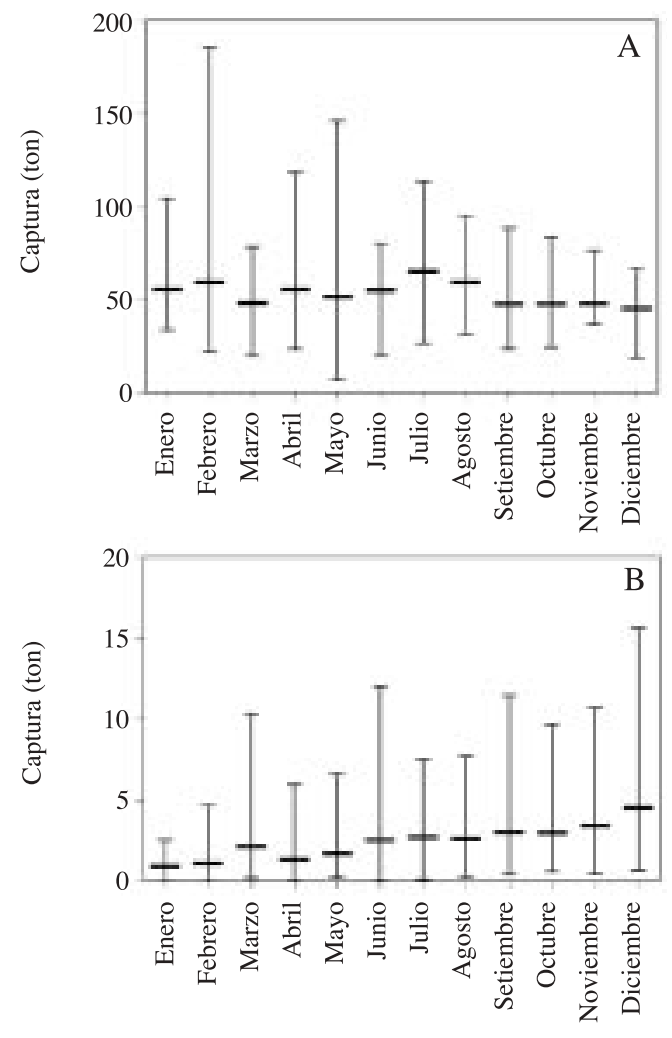

Meses

Fig. 2. Capturas promedio (ton $\boldsymbol{0}$ ), mínimas y máximas ( I) mensuales para el periodo 1984-1994 (t). A: Lutjanus peru. B: Lutjanus guttatus.

Fig. 2. Mean ( $\boldsymbol{E}$ ), minimum, and maximum ( I ) monthly captures (ton) for the period 1984-1994. A: Lutjanus peru. B: Lutjanus guttatus.

Las gráficas de los coeficientes de autocorrelación $(r)$ contra el tiempo para cada especie se presentan en la Fig. 3. En este caso, el número de retrasos está representado en una escala mensual considerando un ciclo bianual. Se aprecia el carácter no estacionario del fenómeno de captura representado por la falta de una atenuación rápida de los valores positivos de autocorrelación.

En el caso de L. guttatus (Fig. 3B) se presentan 7 retrasos con valores positivos de autocorrelación significativamente distintos de 

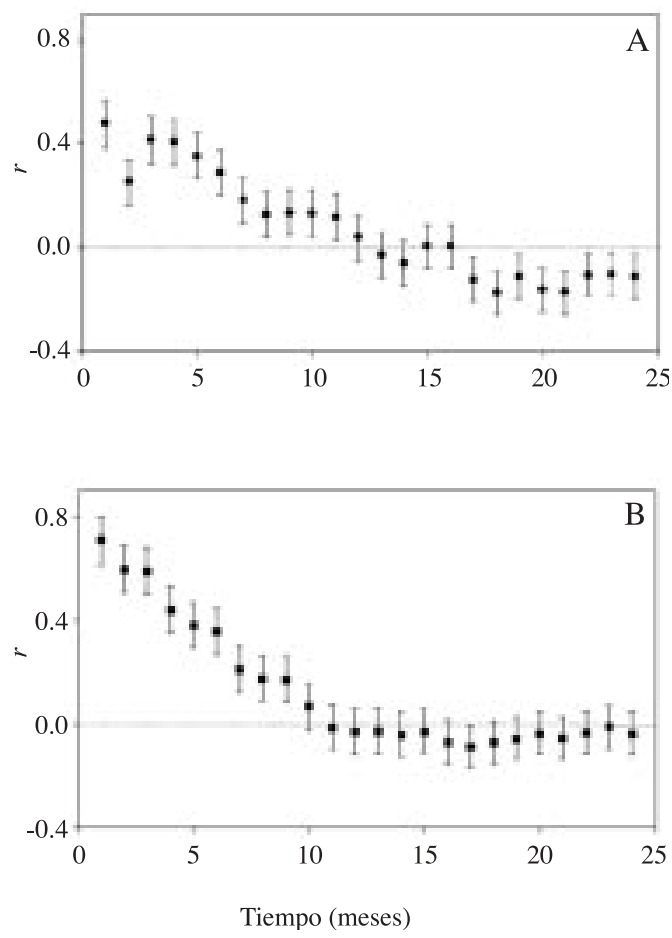

Fig. 3. Coeficientes de autocorrelación $(r)$ de las capturas (ton) versus los retrasos (meses). A: Lutjanus peru $(\bullet \pm$ error estándar). B: Lutjanus guttatus ( \pm error estándar).

Fig. 3. Autocorrelation coefficients $(r)$ of the capture (ton) vs. time lags (months). A: Lutjanus peru $(\bullet \pm$ standard error). B: Lutjanus guttatus ( \pm standard error).

cero $(\mathrm{p}<0.05)$. Si bien el comportamiento de los valores de autocorrelación de $L$. peru es similar (Fig. 3A), la dispersión de los valores positivos es mayor.

El análisis de los resultados de los valores de correlación cruzada $(\rho)$, de los valores de captura de ambas especies (Fig. 4), muestra que la mayor correspondencia entre las dos series de tiempo tiene lugar en el retraso +28 $(\rho=0.56)$. Dado que ambas series tienen un origen común y la misma escala de tiempo, fue posible expresar la posición de las parejas de datos en valores de retrasos positivos y negativos con respecto a la posición original, por lo que es posible afirmar que los valores más altos de captura de ambas especies tienen $\sim 28$ meses de retraso.

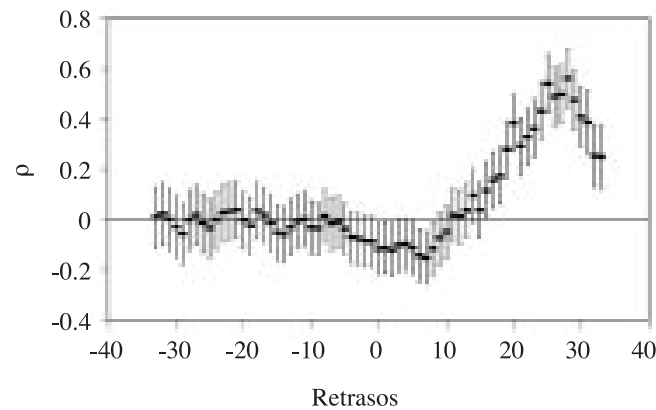

Fig. 4. Coeficientes de correlación cruzada $(\rho \in \pm$ error estándar) de los valores de las series de tiempo de las capturas mensuales (Lutjanus peru y Lutjanus guttatus) vs. los retrasos (meses).

Fig. 4. Monthly capture cross-correlation coefficients

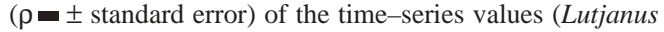
peru and Lutjanus guttatus) vs. time lags (months).

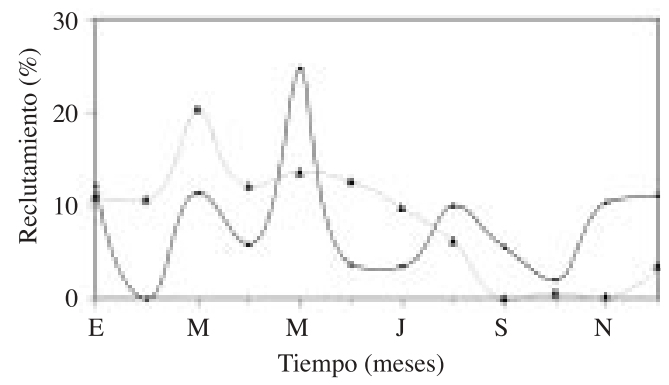

Fig. 5. Patrones anuales de reclutamiento (\%) de Lutjanus peru (- - ) y de Lutjanus guttatus (-)-) obtenidos mediante el paquete FiSAT (Gayanilo et al. 1996). El área bajo las curvas corresponde al $100 \%$ de reclutamiento anual.

Fig. 5. Yearly recruitment pattern (\%) of Lutjanus peru $(-\mathbf{-}-)$ and Lutjanus guttatus (-)-) obtained with FiSAT (Gayanilo et al. 1996). The area under the curve represents the $100 \%$ yearly recruitment.

En la Fig. 5 se presentan los patrones de reclutamiento de $L$. peru y de L. guttatus, obtenidos mediante la aplicación del modelo de Pauly (1987). Si bien ambas especies muestran un reclutamiento continuo, en el caso de $L$. guttatus se observan dos máximos. El primero se produce en el mes de mayo, en el que ocurre el $\sim 25 \%$ del reclutamiento total anual. El segundo, abarca los meses de noviembre a enero (en los que se presentan valores promedio mensuales del $11.2 \%$ ). 
En el caso de L. peru se observa un periodo de máximo reclutamiento ( $\geq 10 \%$ mensual), que comprende los meses de enero a julio, con un valor pico (20\%) en marzo. El periodo de bajo reclutamiento abarca de agosto a diciembre (con un valor promedio mensual $\leq 2 \%)$. Estos resultados indican que ocurre un desfase temporal del reclutamiento de ambas especies. Es interesante notar que los mayores volúmenes de captura para ambas especies (Fig. 2) coinciden con la época de desove estimada.

\section{DISCUSIÓN}

La abundancia de las poblaciones de L. peru y L. guttatus varía mensualmente siguiendo un patrón poco definido que se repite a lo largo del periodo estudiado (Fig. 1). Las fluctuaciones temporales de la abundancia de especies tropicales pueden tener un amplio espectro de causas, desde condiciones que impone el ambiente, incluyendo la disponibilidad del alimento (Stewart y Levin 1973), hasta factores poblacionales, como mortalidad y tasas de reproducción (Madrid et al. 1997). Este conjunto de variables puede operar simultáneamente, teniendo efectos diferenciales, el espacio y el tiempo, sobre la abundancia.

El comportamiento de los valores de autocorrelación de la serie de tiempo de las capturas de $L$. peru presenta una dispersión de valores positivos mayor que los resultados obtenidos para L. guttatus (Fig. 3). Los valores autocorrelativos de corto plazo que se presentan en la Fig. 3 son el producto de la secuencia temporal que persiste en meses sucesivos. La alternativa de análisis para reducir estas variaciones sería la de utilizar los promedios de los valores de las series de tiempo agrupados en intervalos mayores (Tan 2001). En el análisis de los resultados de este trabajo, no se optó por dicha opción puesto que se enmascara la variación temporal en escalas de tiempo mensuales. De esta forma, fue posible relacionar los coeficientes obtenidos con la extensión de la temporada de reproducción, que es mayor para L. peru (Fig. 5).
Los resultados de Saucedo-Lozano et al. (1999), Saucedo-Lozano y Chiappa-Carrara (2000) y Rojas-Herrera y Chiappa-Carrara (2002) indican que existe un gran número de presas comunes a $L$. peru y L. guttatus tanto en la fase juvenil como en la adulta, por lo que se puede inferir que la competencia entre ambas especies constituye una fuerza reguladora de su abundancia poblacional. Para que este mecanismo opere y estas especies coexistan, es necesario que se cumpla la condición de que L. peru y L. guttatus hayan sufrido, a lo largo de su historia evolutiva, diferencias en la morfología, fisiología o rasgos de la historia de vida que reduzcan la competencia y les permitan compartir los recursos alimenticios en el tiempo y/ó el espacio. Sin embargo, los resultados del presente trabajo indican que el mecanismo que favorece la coexistencia de estas especies es el de la separación temporal en los picos máximos del reclutamiento (Fig. 5). Nuestras estimaciones, obtenidas mediante la aplicación del FiSAT, coinciden con las observaciones de Rojas (1997) realizadas para L. guttatus en el litoral de Costa Rica. Este autor reporta que existe un período de reproducción prolongado, con un pico de desove en el mes de abril y otro que abarca de agosto a noviembre.

Si bien existen similitudes en las dietas de estos lutjánidos analizadas globalmente, Rojas-Herrera et al. (2004) encontraron que existen diferencias significativas en los valores de diversidad de la dieta de L. guttatus y de L. pe$r u$. Los valores máximos de amplitud trófica de cada especie reportados por dichos autores, coinciden con los picos máximos del reclutamiento (indicativo de la temporada reproductiva) de estas especies. Asimismo, los valores mínimos de traslape dietético entre estas especies (Rojas-Herrera et al. 2004) ocurren en coincidencia con estos eventos de reclutamiento (Fig. 5). Lo anterior permite postular que entre estas especies la competencia por el alimento no opera como un mecanismo regulador de la abundancia.

Postulados de este tipo han sido propuestos para explicar la coexistencia de otras especies congéneres tanto en climas tropicales 
(Ibáñez-Aguirre 1993, Díaz-Ruiz et al. 2000) como templados (Cardona 2001). En contraposición al punto de vista clásico, de que la redimensión del nicho permite reducir el traslape trófico (Gause 1934, MacArthur y Levins 1967), estos autores consideran que la biología reproductiva de las especies, a través del desfase temporal de los eventos reproductivos y, por lo tanto, del reclutamiento, explica la coexistencia. Los resultados del presente trabajo apoyan esta hipótesis. La resultante de este mecanismo, se ve reflejada en términos de una sucesión temporal de la abundancia de los reclutas de L. peru y L. guttatus en el litoral de Guerrero (Fig. 5).

Otra forma de reducir la competencia interespecífica entre especies que coexisten, sin que ocurra una diferenciación del nicho, ocurre a partir de fluctuaciones en la abundancia poblacional (Jensen 1997). En las especies que están sometidas a explotación pesquera, la mortalidad por pesca puede regular la abundancia poblacional. En este caso, la presión que ejercen las actividades pesqueras sobre las poblaciones de $L$. peru y L. guttatus puede reducir los niveles de competencia interespecífica y favorecer la coexistencia mediante fenómenos de segregación espacio-temporal. Los resultados del análisis de correlación cruzada (Fig. 4) indican que sólo cada 28 meses coinciden los valores más altos de captura de ambas especies, favoreciendo la hipótesis de segregación espacio-temporal. Así, el efecto de la pesca se puede expresar, como un mecanismo de separación del nicho ecológico de las especies de lujánidos, que concurren en la pesquería artesanal del litoral de Guerrero. Jensen (1997) mostró que la variación en la mortalidad de dos especies que presentan valores altos de traslape dietético, define la abundancia relativa de ambas y su capacidad de competir eficientemente por un mismo recurso. De acuerdo con los principios de este mecanismo, es posible suponer que la población de L. peru permite el desarrollo de las otras especies del mismo género que se capturan por la misma pesquería cuando el tamaño de ésta se reduce por debajo de un nivel crítico inducido por la pesca. El sistema de pesca artesanal, provoca un desequilibrio en los valores de abundancia relativa de estos lutjánidos, que persiste debido al incremento en la mortalidad, y da lugar a una consecuente reducción diferencial en el tamaño de cada población.

\section{AGRADECIMIENTOS}

El presente trabajo fue posible gracias al apoyo del CONACYT a través de la Dirección de Asuntos Internacionales dentro del programa de cooperación México-Cuba 1994 y al financiamiento obtenido mediante el proyecto PAPIIT IN211999.

\section{RESUMEN}

Se realizó un análisis de series de tiempo, de autocorrelación y de correlación cruzada, para el estudio de ocho ciclos anuales de variación en los volúmenes de captura de Lutjanus peru y L. guttatus, en la costa de Guerrero, México. Sobre la base de datos de distribución de frecuencias de tallas mensuales, se estimaron los patrones de reclutamiento, empleando el paquete de programas FiSAT. Las capturas de las poblaciones de $L$. peru y $L$. guttatus variaron sin presentar un patrón anual bien definido. Los resultados apoyan la hipótesis de que el desfase temporal de los eventos reproductivos y, por lo tanto, del reclutamiento, explica la coexistencia de estas especies. La resultante de este mecanismo se ve reflejada en términos de una sucesión temporal de la abundancia de los reclutas de $L$. peru y $L$. guttatus en el litoral de Guerrero, lo cual conduce también a una segregación de los reclutas, provocando que el consumo de un mismo tipo de presas ocurra en periodos alternos.

\section{REFERENCIAS}

Álvarez-Lajonchere, L. 1976. Contribución al ciclo de vida de Mugil curema Valenciennes in Cuvier et Valenciennes, 1836 (Pisces, Mugilidae). Rev. Invest. Mar. 28: $1-130$.

Box, G.E.P. \& G. Jenkins. 1976. Time Series Analysis: Forecasting and Control, Holden-Day, California, Estados Unidos.

Butler, A.J. \& A.D.M. Smith. 1992. Effects of recruitment variability on coexistence of competing species: management implications. Proc. Bur. Rural Resour. (Aust.) 16: 157-166. 
Caley, M.J., M.H. Carr, M.A. Hixon, T.P. Hughes, G.P. Jones \& B.A. Menge. 1996. Recruitment and the local dynamics of open marine populations. Annu. Rev. Syst. 27: 477-500.

Cardona, L. 2001. Non-competitive coexistence between Mediterranean grey mullet: evidence from seasonal changes in food availability, niche breath and trophic overlap. J. Fish Biol. 59: 729-744.

Chesson, P.L. 1985. Coexistence of competitors in spatially and temporally varying environments: a look at the combined effects of different sorts of variability. Theor. Popul. Biol. 28: 263-287.

Connell, J.H. 1978. Diversity in tropical rain forests and coral reefs. Science 199: 1302-1310.

Díaz-Ruiz, S., A. Aguirre-León \& O. Pérez-Solis. 2000. Distribución y abundancia de Syngnathus lousianae y Syngnathus scovelli (Syngnathidae) en la Laguna de Tamiahua, Golfo de México. Cien. Mar. 26: $125-143$

Dudgeon, S.R., R.S. Steneck, I.R. Davison \& R.L. Vadas. 1999. Coexistence of similar species in a space-limited intertidal zone. Ecol. Monogr. 69: 331-352.

Elliot, J.K. \& R.N. Mariscal. 2001. Coexistence of nine anemonefish species: differential host and habitat utilization, size and recruitment. Mar. Biol. 138: 23-36.

Evans, S. \& B. Tallmark. 1985. Niche separation within the mobile predator guild on marine shallow soft bottoms. Mar. Ecol. Prog. Ser. 23: 279-286.

Franks, J.S. \& K.E. VanderKooy. 2000. Feeding habits of juvenile lane snapper Lutjanus synagris from Mississippi coastal waters, with comments on the diet of gray snapper Lutjanus griseus. Gulf Carib. Res. 12: 11-17.

Gaines, S. \& J. Roughgarden. 1985. Larval settlement rate: a leading determinant of structure in an ecological community of the marine intertidal zone. Proc. Natl. Acad. Sci. USA 82: 3707-3711.

Gayanilo, F.C.Jr., P. Sparre \& D. Pauly. 1996. The FAO-ICLARM stock assessment tools (FiSAT) user's guide. FAO Computerized Information Series (Fisheries) 8: 1-126.

Gause, G.F. 1934. The struggle for existence. Hafner.

Genner, M.J., G.F. Turner \& S.J. Hawkins. 1999. Foraging of rocky habitat cichlid fishes in Lake Malawi: coexistence through niche partitioning? Oecologia 121: 283-292.

Giller, P.S. 1984. Community Structure and the Niche. Chapman \& Hall.
Guevara, C.E., M.A. Bosch, M.R. Suárez \& R.R. Lalana. 1994. Alimentación natural de tres especies de pargos (Pisces: Lutjanidae) en el Archipiélago de los Canarreos, Cuba. Rev. Invest. Mar. 15: 63-72.

Haight, W.R., J.D. Parrish \& T.A. Hayes. 1993. Feeding ecology of deepwater lutjanid snappers at Panguin Bank, Hawaii. Trans. Am. Fish. Soc. 122: 328-347.

Hassell, M.P. 1986. Detecting density dependence. Trends Ecol. Evol. 1: 90-93.

Ibáñez-Aguirre, A.L. 1993. Coexistence of Mugil cephalus and M. curema in a coastal lagoon in the Gulf of Mexico. J. Fish Biol. 42: 959-961.

Jensen, A.L. 1997. Limiting similarity for coexistence of lake herring (Coregonus artedii) and chubs (Coregonus hoyi). Ecol. Model. 95: 11-15.

Legendre, P. \& L. Legendre. 1998. Numerical Ecology. Elsevier. Amsterdam, Alemania.

MacArthur, R.H 1958. Population ecology of some warblers of north-eastern coniferous forests. Ecology 39: 599-619.

MacArthur, R.H. \& R. Levins. 1967. The limiting similarity, convergence, and divergence of coexisting species. Amer. Nat. 101: 377-385.

Madrid, J., P. Sánchez \& A.A. Ruiz. 1997. Diversity and abundance of a tropical fishery on the Pacific Shelf of Michoacán, México. Estuar. Coast. Shelf Sci. 45: 485-495.

Margalef, R. 1981. Ecología. Omega. Barcleona, España.

Mullon, C., P. Cury \& P. Penven. 2002. Evolutionary individual-based model for the recruitment of anchovy (Engraulis capensis) in the southern Benguela. Can. J. Fish. Aquat. Sci. 59: 910-922.

Paine, R.T. 1977. Controlled manipulations in the marine intertidal zone, and their contributions to ecological theory. Acad. Nat. Sci. Spec. Publ. 12: 245-270.

Pauly, D., J. Ingles \& R. Neal. 1984. Application to shrimp stocks of objective methods for the estimation of growth, mortality and recruitment-related parameters from length-frequency data (ELEFAN 1 and 2), pp. 220-234. In J.A. Gulland \& B.J. Rothschild (eds.). Penaeid shrimps, their biology and management. Fishing News Books, Farnham, Surrey, Inglaterra.

Pauly, D. 1987. A review of the ELEFAN system for analysis of length frequency data in fish and aquatic invertebrates. ICLARM, Contrib. 232: 7-34.

Pfister, C.A. 1992. Sculpin diversity in tidepools. Northwest Environ. J. 8: 156-157. 
Rojas, M.J.R. 1997. Fecundidad y épocas de reproducción del "pargo mancha" Lutjanus guttatus (Pisces: Lutjanidae) en el Golfo de Nicoya, Costa Rica. Rev. Biol. Trop. 44/45: 477-487.

Rojas-Herrera, A.A. 2001. Aspectos de la dinámica poblacional de Lutjanus peru (Nichols y Murphy, 1922) y L. guttatus (Steindachner, 1869) (Pisces: Lutjanidae) del litoral de Guerrero, México. Tesis doctoral, Universidad de Colima, $194 \mathrm{p}$.

Rojas-Herrera, A.A. \& X. Chiappa-Carrara. 2002. Feeding habits of the spotted rose snapper Lutjanus guttatus (Pisces: Lutjanidae) in the coast of Guerrero, Mexico. Cienc. Mar. 28: 133-147.

Rojas-Herrera, A.A., M. Mascaró \& X. Chiappa-Carrara. 2004. Hábitos alimentarios del huachinango (Lutjanus peru) y del flamenco (Lutjanus guttatus) (Pisces: Lutjanidae) en la costa de Guerrero, México. Rev. Biol. Trop. 52: 163-170.

Sámora-Zapata, J.C. \& M.E. Vega-Cendejas. 1998. Ecología alimenticia e interacción trófica de los pargos Lutjanus griseus (Linnaeus, 1758) y Lutjanus synagris (Linnaeus, 1758) de la laguna de Celestum, Yucatán, México, pp.15. In Memorias del VI Congreso Nacional de Ictiología. Universidad Veracruzana. Tuxpan, Veracruz, México.

Saucedo-Lozano, M., G. González-Sansón \& X. ChiappaCarrara. 1999. Natural feeding of juveniles of Lutjanus peru (Nichols and Murphy, 1922) (Lutjanidae: Perciformes) off the coast of Jalisco and Colima, Mexico. Cienc. Mar. 25: 381-400.
Saucedo-Lozano, M. \& X. Chiappa-Carrara. 2000. Alimentación natural de juveniles de Lutjanus guttatus (Pisces: Lutjanidae) en la costa de Jalisco y Colima, México. Bol. Cent. Invest. Biol. (Maracaibo) 34: 159-180.

Sierra, L.M. 1997. Relaciones tróficas de los juveniles de cinco especies de pargos (Pisces: Lutjanidae) en $\mathrm{Cu}-$ ba. Rev. Biol. Trop. 44/45: 499-506.

Sinclair, A.R.E. 1989. Population regulation in animals, pp. 197-241. In J.M. Cherret (ed.). Ecological Concepts: the contribution of Ecology to an understanding of the natural world. Blackwell Londres, Inglaterra.

Stewart, F.M. \& B.R. Levin. 1973. Partitioning of resources and the outcome of interspecific competition: a model and some general considerations. Amer. Nat. 107: 171-198.

Tan, P.N., M. Steinbach, V. Kumar, C. Potter, S. Klooster \& A. Torregrosa. 2001. Finding spatio-temporal patterns in earth science data, pp. 1-12. In Proceedings of the $7^{\text {th }}$ International Conference on Knowledge Discovery and Data Mining.

Vega-Cendejas, M.E. 1998. Trama trófica de la comunidad nectónica asociada al ecosistema de manglar en el litoral norte de Yucatán. Tesis doctoral, Facultad de Ciencias, UNAM, México. 170 p.

Visauta-Vinacua, B. 1998. Análisis estadístico con SPSS para Windows (Volumen II Estadística multivaríante). McGraw-Hill. Madrid, España. 
